\title{
PREDICTORS OF A CHILD'S SOCIAL SKILLS ASIT RELATES TO MOTHER ATTACHMENT STYLES AND MOTHER ANXIETY LEVELS AMONG STUDENTS IN GRADES (1-3)
}

\begin{abstract}
Khaeld Shaker Alsaraireh
Department of Psychology, Faculty of Education, Al-Balqa Applied University, Jordan

Received 2013-03-19, Revised 2013-05-03; Accepted 2013-05-04

ABSTRACT

The aim of this study was to find the predictors of a child's social skills from mother attachment styles and anxiety levels among students in (1-3) grades in Amman city. A sample of (250) mothers for students in (13) grades in Amman city were selected through purposive sampling methods. Three instruments were used (Attachment Style Questionnaire, Social Skills Rating System (SSRS) and Trait Anxiety Inventory) to measure the study variable. The study results revealed that most of the attachment styles were consistent and there was a wide range of attachment styles that were common among mother of school children. The children's social skills were found to be in the moderate level and acceptable. The mother's anxiety levels insinuate a high level of anxiety in terms of either her state or anxiety traits. The multiple regression analysis revealed that there were four predictors of mother attachment style that had a significant impact on a child's social skills. These predictors included, "confidence", "need for approval", "preoccupation with relationships confidence" and "relationships as secondary". All the aforementioned predictors were noted to have a positive impact on social skills. Finally, the mother's anxiety level, more specifically the anxiety state or traits had a significant negative impact on social skills development of a child.
\end{abstract}

Keywords: Child Social Skills, Mother Attachment Styles, Mother's Anxiety

\section{INTRODUCTION}

According to Bowlby (1973), securely attached child uses the attachment figure as a safe haven to explore the surrounding environment and then returns to this attachment figure during times of distress. The child's experiences with attachment figures such as their caregivers assist in their ascertaining the availability of their caregivers. Children who have a secure attachment to their caregivers perceive their caregivers as caring, responsive and available. In contrast, children who have insecure attachment do not feel safe with their attachment figure (s) nor do they respond positively to interactions with the attachment figure. Uncertainty about the availability of attachment figures further increases a child's likelihood in responding with fear and anxiety when experiencing threatening situations. Subsequently, the child or "the person concerned is often referred to as suffering from free-floating anxiety" (Rutter, 2008).

Research has shown that a child's attachment to their mother plays a vital role in developing and improving their social skills when interacting with peers (Verschueren and Marcoen, 2005). Furthermore, many research findings recommend additional exploration be undertaken in examining the relationship between a child's social skills and their attachment with their mother. The childhood stage is one where the child can experience positive feelings such as joy and one where personality formation begins in where psychological and social skills are developed. Also the relationship between the child and their mother help shape the main characteristics of the child's psychological and social traits in the future. However for some children, making friendship and engaging in social activities with other children is a source of tremendous stress. A child who 
experiences distress when interacting with other children or adults is more likely to avoid social situations.

In order to foster desirable social skills in a child, a child needs to feel a sense of security during the early stages of their lives, more specifically during the developmental period where they are exploring their social environments. The primary caregiver is the main source in nurturing these positive social skills in a child through the exemplifying of a secure attachment style. The quality of attachment between child and mother is one factor that may influence the feeling of safety and social anxiety (Vertue, 2003) in play and peer interaction which ultimately can hinder the child's development of social skills.

A review conducted by Hudson and Rapee (2001) on the topic of a child's attachment with their parents suggest that the father-child attachment in particular was correlated to social anxiety disorders in children. In contrast, the mother-child attachment was acknowledged as being the primary model for a one to one relationship that could help best address separation anxiety issues. It is presupposed that secure mother-child attachment will protect against the development of separation anxiety disorder in children (Hudson and Rapee, 2001).

Weems and Costa (2005) proposed a model of association between maternal and child anxiety. The model posits that maternal anxious attachment beliefs were an important component which mediated the association between maternal and child anxiety. An anxious mother-child relationship is established where maternal anxiety is related to maternal anxious attachment beliefs. This finding is consistent with research on parenting and attachment which has consistently shown that children with perceived negative parenting and early insecure attachment relations are significantly more likely to develop psychopathology, including both emotional and behavioral problems. Similarly, part of the mother psychological characteristics may be passed on to the child such as the mother-anxiety level may transfer to the child and influence the child's capacity of social skills.

In addition, Eapen et al. (2007) conducted a study in the United Arab Emirates aimed at finding the relationship between social anxiety disorders and feeling of shyness in early childhood. A sample of (200) children aged five years and their mothers was taken. This sample measured for shyness in children (Stevenson (1994) and the mother social anxiety scale was used to measure social anxiety among mothers for (Liebowitz, 1972). Results showed that $27 \%$ of shy children in early childhood have been associated shyness with same- gender parent. Results also indicate that there is a relationship interaction between maternal anxiety and the child's shyness in pre-school stage.

Additionally, Simons et al. (2001) studied the relationship between the quality of attachment of adolescents with parents and manifestations of aggression emerging during adolescence. Results showed that high levels of social knowledge among teenagers have been associated with positive and statistically significant outcomes. In fact, the findings showed that high levels of social knowledge correlated with lower levels of reports of aggression by teenagers. The findings also identified the level of attachment outcome influenced the child's normal growth, especially in the emotional and social spheres. There is ample evidence that can be drawn from Western literature that indicates the mother attachment style and the mother's level of anxiety impact the development of a child's social skills. The limited research of this topic in Arab countries further validates the need for additional research studies on this topic. The purpose of this study was to define and measure the impacts of some emotional characteristics of the mother and the family structure on a child's social skills development.

\subsection{Study Problem}

An anxious mother will limit the scope of social interaction through interruption and repetitive inhibition for a child who participates in group playing activity because insecure attachment between the mother and the child diminishes a child's social interaction capacity. Also, the relationship between attachment and anxiety was confirmed in Arabic literature. For example, AlHirot (2000) investigated the relationship between patterns of attachment to the mother in late childhood and social anxiety during childhood. The results were consistent and confirmed the presence and impacts of attachment patterns on social anxiety. Moreover, there was no significant relationship between attachment patterns and gender on social anxiety. This is an important consideration to taken, as physical and emotional contact between the mother and the child in the Arab culture is encouraged and displayed outwardly. However, this does not always portray the actual circumstance and healthiness of the mother-child relationship, thus this study is trying to find the impact of mother attachment and anxiety, on a child social skills, in more specific team the scope of this study will focus on answering the following questions:

- What is the attachment type that predicts the social skills of students in (1-3) grades in Amman 
- What is the mother-anxiety that predicts the social skills of students in (1-3) grades in Amman

- What are the impacts of the family structure variables (number family member, birth order and child gender) on a child's social skills among students in (1-3) grades in Amman

\section{MATERIALS AND METHODS}

\subsection{Research Design}

The researcher employed the descriptive research design of the survey type. This plan of study is considered appropriate because it focuses on the observation and perception of the existing situation.

\subsection{Population}

The population for the study is comprised of school aged children in grades (1-3) who attend private schools in Amman city and also includes their mothers. During the first semester in the academic year 2012-2013, the study population estimated approximately (20141) students.

\subsection{Sample and Sampling Procedure}

Two hundred and fifty children and their mothers were selected for this study through the purposive sampling technique. Six big private schools were randomly selected from a list of the private schools which included grades (1-3), 144 (57.6\%) of whom were females and $106(42.2 \%)$ were males. Also, 98 (39.2\%) students were from grade one, 87 students (34.8\%) from second grad and 65 students (26\%) from the third grade. Also, the means of family member 3.75 with STD 1.25 and the means of the birth order 2.41 with STD 0.95 .

\subsection{Study Instruments}

\subsubsection{Attachment Style Questionnaire}

The ASQ Feeney et al. (1994) is a 40-item self-report which examines bidirectional effects of attachment, Each ASQ item is rated on a 5-point Likert-scale. The respondent is asked to "indicate how much you disagree or agree with each of the following statements" by circling $1=$ strongly disagree, $2=$ disagree, $3=$ moderate agree, $4=$ agree, $5=$ strongly agree.

The negative items of the questionnaire are reversed before they were tabulated to calculate the total scores for each subscale. All the subscale scores range from (15) to find a similar maxim score in order to make a comparison between the subscale scores. The high score of 3 and above implies that the person identifies more closely with the attachment type.

The SSRS has high reliability and validity indices for use with children. The psychometric properties of the SSRS indicated that alpha reliability coefficient of the social skills scale was 0.90 . Test-retest reliability scores at 4 weeks were reported between 0.65 and 0.87 (Gresham and Elliot, 1990). The questionnaire measure classifies adult attachment style on five factors; (a) confidence, (b) discomfort with closeness, (c) need for approval, (d) preoccupation with relationships and (e) relationships as secondary.

\subsection{Social Skills Measure}

Social skills were measured by using the Social Skills Rating System (SSRS). This system was developed by Gresham and Elliot (1990) and was used in this study to obtain information on the child social behavior. The SSRS uses ratings based on frequency to reflect how often a social behavior occurs. The frequency is measured on a five-point scale ranging from 'never' to 'very often.' Scores range from 1 to 5 after and the 'mean' of the child's score is calculated by tabulating the sum and then divide the sum by the total number of items. In summary, higher scores indicate a behavior that occurs frequently and is indicative of the child having more social skills.

\subsection{Trait Anxiety Inventory}

The Trait Anxiety Inventory was used. The inventory composed from (20) items is an administered analysis of reported anxiety symptoms. The first subscale measures state anxiety, while the second measure trait anxiety. The range of scores is $1-5$. The sum of the total response is divided by the item numbers and the higher the scores, so too is the level of anxiety (Rule and Traver, 1983). some of the questions relate to the absence of anxiety and are reversed-scored.

Scale validity on its origin indicts shows that it has construct validity. This is determined by calculating the correlation by its scores and with the Panic Disorder and right-hemisphere brain over activation (Smeets et al., 1997).

The Anxiety Scale Questionnaire (ASQ) and Manifest Anxiety Scales (MAS) have a positive correlation of scores $(0.73$ and 0.85$)$ with the STAI -T, which is close enough to show reliability but different enough to be useful in its. The internal reliability of this scale (Chronbach's alpha) ranges from 0.81-0.86 with retest reliability at a 2-week anxiety determination (Spielberger et al., 2002). 
Khaeld Shaker Alsaraireh / Journal of Social Sciences 9 (1): 22-28, 2013

Table 1. Multiple regressions for the attachment types of the mothers, mother's anxiety predicting children social skills

\begin{tabular}{lllllll}
\hline Model & $\mathrm{R}$ & $\mathrm{R}$ & $\begin{array}{l}\text { Adjusted } \\
\text { S Square }\end{array}$ & $\begin{array}{l}\text { Std. Error of } \\
\text { the Estimate }\end{array}$ & F & Sig. \\
\hline attachment types & 0.55 & 0.30 & 0.29 & 0.35 & 52.21 & $0.00^{*}$ \\
Anxiety & 0.44 & 0.19 & 0.16 & 0.40 & 18.42 & $0.00^{*}$ \\
Family structure & 0.60 & 0.36 & 0.30 & 0.42 & 64.24 & $0.00^{*}$ \\
\hline
\end{tabular}

Significant at level 0.05 or less

\subsection{Scale Reliability}

Test-retest reliability of the STAI was evaluated using 29 male undergraduate students before and after a stressful social analogue situation (Rule and Traver, 1983). The first test administration occurred approximately two weeks before the stressful event and the retest was administered after the analogue social situation. The study results supported previous studies using the STAI in which state anxiety increased from the test to the retest while the trait anxiety remained at similar levels before and after (Rule and Traver, 1983).

\subsection{Family Structure Variables}

A number of family structure variables were obtained from mothers of the participating children such as number of family member, birth order and child gender in order to answer the third study question.

\subsection{Data Collection Procedure}

In order to achieve study goals the following steps were used:

- Translate the study instrument to the Arabic language

- The back translation procedure was applied to study instruments in order to verify its translation validity

- Validity and reliability were conducted for the instrument to make sure that it has the appropriate psychometric indices

- Six schools were randomly selected to administrate study instrument on it

- Study instrument was sent to 380 child mother to complete it, the returned number of study instruments were (250)

\subsection{Data Analysis Procedure}

To analysis the study data, descriptive statistics were used such as a mean standard deviation, besides, multiple linear regression analysis was conducted to find out the prediction value of the independent variables (attachment style, anxiety) of the dependent variable (social skills).

\subsection{Study Results}

This study was embarked upon for the purpose of finding the predictors of a child's social skills in relation to mother attachment styles, mother anxiety level and family structure among students in (1-3) grades in Amman city. The result of question one: "What is the attachment type that predicts the social skills of students in (1-3) grades in Amman?" Table 1 indicated the multiple $\mathrm{R}$ coefficients for mother attachment style was relatively high. In others words, the findings imply that there is a significant effect of the predicting factors --that is that they had a positive impact on social skills.

As is noted in Table 1, the multiple R coefficients for mother attachment style is $(0.55)$ and the (F 52.21, D.F. 5244 , Prob $\leq 0.01)$, which indicates a significant effect of the predicting variables and dependent variables (child social skills). The $\mathrm{R} 2$ value of 0.30 that part of the variance of social skills was explained by one or more attachment style.

The result in Table 2 shows that only four predictors of mother attachment style had a significant impact on child social skills. The first one was the 'Confidence' $(\beta$ $=0.24, \mathrm{t}=4.12$, Prob $\leq 0.01)$. The second one was 'need for approval' $(\beta=0.33, t=4.38$, Prob $\leq 0.01)$. The third variable was 'preoccupied with relationships confidence' $(\beta=-0.19, \mathrm{t}=2.83$, Prob $\leq 0.01)$. Finally, the forth variable was 'relationships as secondary' $(\beta=0.17, \mathrm{t}=$ 3.17, Prob $\leq 0.01)$. All the predicting factors had a positive impact on social skills.

\subsection{Question Two}

What is the mother-anxiety that predicts the social skills of students in (1-3) grades in Amman? To answer this question, entry multiple regressions were conducted to find the predicting value for the mother's anxiety. As is noted in Table 1, the multiple $\mathrm{R}$ coefficients for mother's anxiety is (0.44) and the (F 18.42, D.F. 2247 , Prob $\leq 0.01$ ) which indicates a significant effect of the predicting variables on the dependent variables (child social skills). The $\mathrm{R} 2$ value $=0.193$ indicates part of the variance of social skills was explained by one or more of mother anxiety level. Furthermore, multicollinearity test showed that the VIF values of all the predicting variables were less than (5). 
Khaeld Shaker Alsaraireh / Journal of Social Sciences 9 (1): 22-28, 2013

Table 2. Standardized Coefficients for the attachment types of the mothers for children, mother anxiety and family structure variables social skills

\begin{tabular}{|c|c|c|c|c|c|c|}
\hline & \multicolumn{2}{|l|}{ Unstandardized coefficients } & \multicolumn{4}{|c|}{ Standardized coefficients } \\
\hline & Model & $\mathrm{B}$ & Std. Error & Beta & $\mathrm{t}$ & Sig. \\
\hline \multirow[t]{6}{*}{ Attachment types } & (Constant) & 0.89 & 0.14 & & 6.56 & $0.00 *$ \\
\hline & Confidence & 0.21 & 0.05 & 0.24 & 4.12 & $0.00 *$ \\
\hline & Discomfort with closeness & 0.00 & 0.06 & 0.00 & 0.05 & 0.96 \\
\hline & Need for approval & 0.29 & 0.07 & 0.33 & 4.38 & $0.00 *$ \\
\hline & Preoccupation with relationships. & 0.17 & 0.06 & 0.19 & 2.83 & 0.01 \\
\hline & Relationships as secondary & 0.14 & 0.05 & 0.17 & 3.17 & $0.00 *$ \\
\hline \multirow[t]{3}{*}{ Anxiety } & (Constant) & 0.31 & 0.19 & & 1.66 & 0.10 \\
\hline & Stat & 0.62 & 0.09 & -0.30 & -7.27 & $0.00 *$ \\
\hline & Trait & 0.30 & 0.09 & -0.27 & -3.43 & $0.00 *$ \\
\hline \multirow[t]{3}{*}{ Family structures } & number of family member & 0.39 & 0.03 & 0.44 & 11.39 & $0.00 *$ \\
\hline & Birth order & 0.35 & 0.06 & 0.27 & 5.64 & $0.00 *$ \\
\hline & Child gender & 0.28 & 0.06 & 0.21 & 4.44 & $0.00 *$ \\
\hline
\end{tabular}

Significant at level 0.05 or less

In order to find out the Standardized Beta Coefficients, it is necessary to determine which one gives a measure of the contribution of each variable to the model? The result in Table 2 shows that the two predictors of mother's anxiety had a significant impact on a child's social skills. The first one was 'anxiety as a state' $(\beta=-0.30-, \mathrm{t}=-7.26$, Prob $\leq 0.01)$ and the second one was 'anxiety as a trait' $(\beta$ $=-0.265-, \mathrm{t}=-3.434$, Prob $\leq 0.01)$. Both calculations provide a measure of the contribution of each variable to the model and showed a negative impact of the mother anxiety on the child social skills.

Question three: What are the impacts of the family structure variables (number family member, birth order and child gender) on a child's social skills among students in (1-3) grades in Amman?

To answer this question, entry multiple regressions were conducted to find the predicting value for family structure variables for children social skills and Table 1 shows the result. As is noted in Table 1, the multiple $\mathrm{R}$ coefficients for family structure is $(0.602)$ and the $(F$ 64.24, D.F. 2 247, Prob $\leq 0.01$ ). These results indicate a significant effect of the predicting variables on the dependent variables (child social skills). The $\mathrm{R} 2$ value $=$ 0.362 means part of the variance of social skills was explained by one or more of family structure variables. Furthermore, multicollinearity test showed that the VIF values of all the predicting variables were less than (5). In order to find out the Standardized Beta Coefficients, which one gives a measure of the contribution of each variable to the model? The result in Table 2 shows that the two predictors of family structure variables had a significant impact on child social skills. The first one was the number of family members $(\beta=0.444, \mathrm{t}=$
11.38, Prob $\leq 0.01)$ and the second variable was child birth order $(\beta=0.273, t=5.64$, Prob $\leq 0.01)$. Also, child gender had a significant beta coefficient $(\beta=0.214, \mathrm{t}=$ 4.43 , Prob $\leq 0.01)$.

\section{RESULTS AND DISCUSSION}

There were three study questions that were tested in this study. The results from the first question revealed that there was a significant effect of the predicting factors --- that is that they had a positive impact on social skills.

These results were confirmed by Hudson and Rapee (2001) who emphasized the important relationship between the parent attachment style and anxiety disorder. Also, these results were partially supported by Eapen $e t$ al. (2007) who found that parent attachment style helped predict the level of shyness among their chidden.

From a practical standpoint, uncovering social skill deficits related to attachment style may be an important step in breaking the cycle of negative reinforcement that some insecure mothers exhibit when they observe their children interacting socially with others. For instance, secure mothers will encourage their children to interact in social situations. Insecure mothers on the other hand will discourage their children in engaging in a social situation. The result of discouraging children in engaging in social activities is that this will limit the opportunity for social skills development.

If a secure and attached child engaged in social situations, their feelings of security was more likely to be reinforced when others positively respond to them (Bartholomew, 1993). In the same context, if insecure individuals engage or observe incompetent social 
situation, their insecurity is likely to be reinforced when others negatively respond to them. Moreover, the result of the question related to the impact of mother anxiety on the child social skills helps provide a better understanding of child social skills and mother-anxiety. A possible reason might be that high anxious mothers may inhibit their children in engaging in social environments because of their own personal fear and the negative expectations they carry inside of them about social situations. Essentially, such mothers may instill in themselves expectations of rejection and ignorance which in turn is projected upon their children. By anxious mothers seeking compensation for their lack of security by limiting and hampering their children's social interaction, they inadvertently negatively influence the child's opportunity to learn and develop functional social skills.

The results to the question "What are the impacts of the family structure variables (number family member, birth order and child gender) on child social skills among students in (1-3) grades in Amman" was interesting as well. The increase of family members in a family impacted the child social skills positively. A possible explanation of this might be that there is greater opportunity of interaction of a child with other family members. This is especially the case in Arab culture, as many families have children that are close to each other in age.

The child birth order may also play a role in a child's social skills development.

The experience attained from raising the first child often allows mothers to be more flexible in their parenting style for their next child by giving them more space for social interaction with peers. This further lends strength to the notion that knowledge and realistic expectation has an important role in a child's social interaction.

In contrast to the result that indicates that there is a significant impact of child gender on the social skills, AlHirot (2000) found no significant relationship between children gender and social skills. From a cultural perspective, this is especially the case in the Arab culture where females who have a close relationship to their mother are often seen to participate in social events with their mother.

Based on the study result, the researcher suggests the following recommendation:

- It is important to increase awareness in anxious mother's about the possible negative impacts anxiety can have on their children's social skills. This can be achieved through teaching parenting skills for both mothers and fathers
- It is important for mother and fathers to share the child raising responsibilities in order that they set realistic expectation for the mother's social and psychological role

- Future research should be conducted in order to better understand the impacts of father anxiety level and attachment type on children's development of social skills

- One possible limitation of this study is sample representation, as the selection was taken from only private schools in Amman. Hence, it is important to conduct further research to include people from public schools whom represent a large population who are from relatively low to moderate socioeconomic cultural background.

\section{CONCLUSION}

The study tried to find the impact of three possible variables in child social skills, the result funding revealed that the mother attached style plays significant role in developing the child social skills, the positive mother aspect of attachment contribute positively in increasing the healthy social skills among their children Also the result of the study confirmed that mother anxiety has a negative role in developing a healthy and functional social skills by mother restriction of child social interaction in social situation. Furthermore, the increase of family members in a family impacted the child social skills positively, as well as the child birth order may also play a role in a child's social skills development, this can be seen in according to Arab culture. In additional, to child gender played important role in developing social skills, female child benefit from her company to her mother to develop a healthy social skills.

\section{REFERENCES}

Al-Hirot, J., 2000. The relationship between patterns of attachment to the mother in late childhood and social anxiety during childhood. Unpublished Mater Thesis, University of Jordan.

Bartholomew, C.G., 1993. Christians and the Arts in South Africa: A Manifesto. 1st Edn., Christian Worldview Network, Wynberg, ISBN-10: 0620189932, pp: 15.

Bowlby, J., 1973. Attachment and Loss: Separation: Anxiety and Anger. 2nd End., Basic Books, New York, pp: 456. 
Eapen, V., A.A. Mabrouk, T. Zoubeidi and F. Yunis, 2007. Prevalence of pervasive developmental disorders in preschool children in the UAE. J. Trop. Pediatr., 53: 202-205. DOI: 10.1093/tropej/fml091

Feeney, J.A., P. Noller and M. Hanrahan, 1994. Assessing adult Attachment. In: Attachment in Adults: Clinical and Developmental Perspectives, Sperling, M.B. and W.H. Berman (Eds.), Guilford Press, New York, ISBN-10: 0898625475, pp: 128154.

Gresham, F.M. and S.N. Elliott, 1990. Social Skills Rating System: Manual. 1st Edn., American Guidance Service, Circle Pines, pp: 194.

Hudson, J.L. and R.M. Rapee, 2001. Parent-child interactions and anxiety disorders: An observational study. Behav. Res. Therapy, 39: 1411-1427. DOI: 10.1016/S0005-7967(00)00107-8

Liebowitz, H., 1972. Fracture, an advanced treatise. 1st Edn., Academic Press, New York London, pp: 1044.

Rule, W.R. and M.D. Traver, 1983. Test-retest reliabilities of state-trait anxiety inventory in a stressful social analogue situation. J. Personality Assess., $\quad 47$ : 276-277. DOI: 10.1207/s15327752jpa4703_8

Rutter, M., 2008. Implications of Attachment Theory and Research for Child Care Policies. In: Handbook of Attachment: Theory, Research and Clinical Applications, Cassidy, J. and P.R. Shaver (Eds.), Guilford Press, New York, ISBN-10: 160623028X, pp: 958-974.

Simons, K.J., C.E. Paternite and C. Shore, 2001. Quality of parent/adolescent attachment and aggression in young adolescents. J. Early Adolescence, 21: 182203. DOI: $10.1177 / 0272431601021002003$
Smeets, G., H. Merckelbach and E. Griez, 1997. Panic disorder and right-hemisphere reliance. Anxiety Stress Cop.: Int. J., 10: 245-255. DOI: 10.1080/10615809708249303

Spielberger, C.D., E.C. Reheiser, L.M. Ritterband, S.J. Sydeman and K.K. Unger, 2002. Assessment of Emotional States and Personality Traits: Measuring Psychological Vital Signs. In: Clinical Personality Assessment: Practical Approaches, Butcher, J.N. (Ed.), Oxford University Press, New York, ISBN10: 0195142586.

Stevenson, F.J., 1994. Humus Chemistry: Genesis, Composition, Reactions. 2nd Edn., John Wiley and Sons, ISBN-10: 0471594741, pp: 496.

Verschueren, K. and A. Marcoen, 2005. Perceived Security of Attachment to Mother and Father: Developmental Differences and Relations to selfWorth and Peer Relationships at School. In: Attachment in Middle Childhood, Kerns, K.A. and R.A. Richardson, (Eds.), Guilford Press, New York, ISBN-10: 1593851219, pp: 212-230.

Vertue, F.M., 2003. From adaptive emotion to dysfunction: An attachment perspective on social anxiety disorder. Pers. Soc. Psychol. Rev., 7: 170191. PMID: 12676646

Weems, C.F. and N.M. Costa, 2005. Developmental differences in the expression of childhood anxiety symptoms and fears. J. Am. Acad. Child Adolescent Psychiatry, 44: 656-653. DOI: 10.1097/01.chi.0000162583.25829.4b 